\title{
Industrial IoT Smartbox for the Shop Floor
}

Sérgio Malhão

Instituto Politécnico de Castelo Branco

Castelo Branco, Portugal

smalhao@ipcbcampus.pt
Rogério Dionísio

Instituto Politécnico de Castelo Branco

Castelo Branco, Portugal

rdionisio@ipcb.pt
Pedro Torres

Instituto Politécnico de Castelo Branco

Castelo Branco, Portugal

pedro.torres@ipcb.pt

\begin{abstract}
Constant search for efficiency and productivity has led to innovation on the factory shop floor, representing an evolution of the current production systems combined with new technologies of industrial automation and information technology. This work presents an experimental demo of a smartbox for Industry 4.0 scenarios, allowing sensing, monitoring and data acquisition. We have tested two different approaches, depending on the communication protocol used for real time applications: OPC UA or MQTT. Raspberry Pi's platform act as an OPC UA server or MQTT broker, respectively. From the measurements, data stored in a cloud server can be accessed remotely with improved security and visualized from a computer dashboard. One of the conclusions that can be drawn is that both protocols allow data from the smartbox to be stored and easily monitored from a smartphone application or a computer web interface. MQTT is a good option in communications requiring very low bandwidth. However, there is a lack of suitable libraries to program alarm features for OPC UA Servers.
\end{abstract}

Keywords-Industrial IoT, OPC UA, MQTT, Raspberry PI, Node-RED

\section{INTRODUCTION}

Recent years witnessed the forthcoming of Machine-toMachine (M2M) networks as an efficient means to provide automated communications between distributed devices. The idea of Smart Industry is gaining increasing importance in the current context because of its ability to automate industrial environments with great effectiveness using Smart systems. These systems incorporate detection, actuation and control functionalities.

The Portuguese industrial sector follows this trend and searches to technologically re-adapt its production processes to include sensing, monitoring and control functionalities, allowing efficient communication with the equipment on the shop floor with Industrial Internet of Things (IIOT) platforms [1].

This paper focuses on the development and testing of a Smartbox demonstrator, which allows sensing, monitoring and storage of equipment parameter's data from industrial environments through Wireless and Ethernet networks, using OPC UA (OPC Unified Automation), Message Queuing Telemetry Transport (MQTT) protocols.

\section{Protocols}

\section{A. $M Q T T$}

MQTT, Created by IBM and by Eurotech, is a standard detailed by the MQTT v3.1 specification [2].

This work is funded by the Portuguese programme PORTUGAL 2020 , under grant agreement $n .^{\circ} 024541$ (PRODUTECH SIF - Soluções para a Indústria do Futuro)

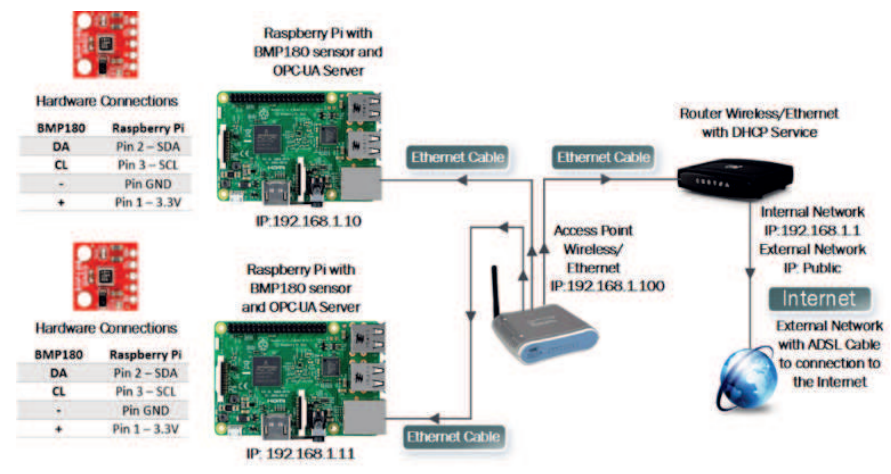

Fig. 1 Communication with OPC UA protocol in a local network.

This communication standard follows a Publisher / Subscriber model, where a Publisher publish information to the Broker (Middleware). The Broker is responsible for receiving and centralizing messages from one or more Publishers, and to dispatch information to any authorized Subscriber requesting access to it, using applications for both $\mathrm{PC}$ and Smartphones.

\section{B. OPC UA}

OPC UA is a standard for communication and information modeling in industrial automation [3]. The specifications are standardized by IEC 62541. This standard allows the implementation of servers in embedded systems. This communication standard can use two communication models, the Request / Reply model and the Publisher / Subscriber model. Messages can be sent in binary format + TCP or Extensible Markup Language (XML) + HTTP / Simple Object Access Protocol (SOAP).

\section{Prototype DeVElopment}

\section{A. Implementation with OPC UA}

The OPC UA demonstrator's architecture is built upon Ethernet and Wi-Fi networks, as can be seen in Fig. 1. For the Raspberry Pi's to act as OPC UA servers, several required libraries were previously installed [4].
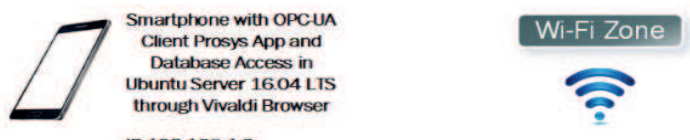

IP 19216813

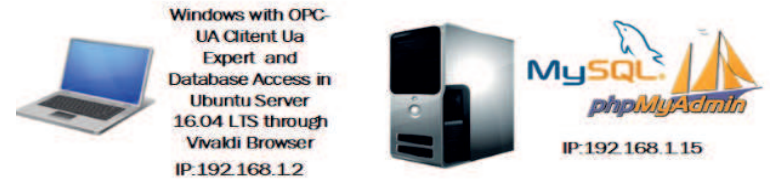

Fig. 2 Software and Hardware to subscribe and store OPC UA data through a WiFi network. 


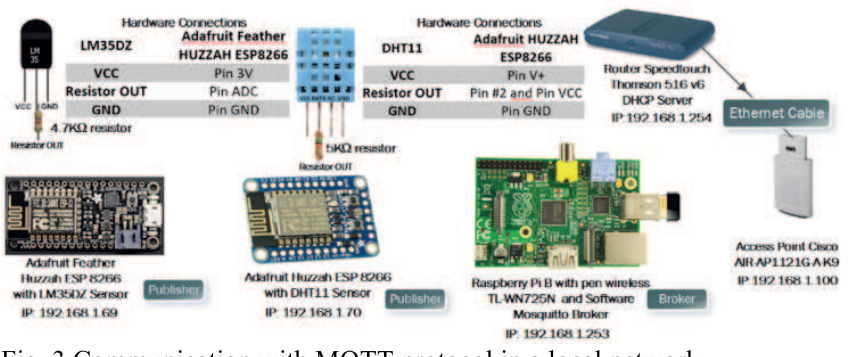

Fig. 3 Communication with MQTT protocol in a local network.

A PYTHON script was written to enable the acquisition of three environmental parameters (temperature, altitude and pressure) available in each BMP180 sensor, through the OPC UA Servers. It was also necessary to set up a set endpoint consisting of an IP address, communication port, library and class, and also to define the object parameters, such as temperature, altitude and pressure, as well as access and permission rules of the OPC UA Clients.

To subscribe and store all data sent from the OPC UA server, we install a MySQL database on UBUNTU Server 16.04 LTS together with Unified Automation UA Expert software [5] on a WINDOWS 10 virtual machine, as shown in Fig. 2. On the Client side, we use a PROSYS Smartphone application [6] to subscribe to the sensor data parameters.

\section{B. Implementation with $M Q T T$}

The network architecture presented in Fig. 3, demonstrates data acquisition using MQTT protocol [2]. Two ESP8266 modules were configured as Publishers and connected to a temperature sensor (LM35DZ) and a humidity/temperature sensor (DHT11). Topics (data) are sent from Publishers to a Broker (MOSQUITO) [7] installed on a Raspberry $\mathrm{Pi}$ platform. All authorized Subscribers can subscribe to the Broker platform for Topics; for the LM35DZ module the topics is "esp8266" and for the DHT11 module, the topics are "dht11humidity" and "dht11 temperature".

Each Publisher module has received a script in $\mathrm{C} / \mathrm{C}++$ with network SSID and authentication credentials to access the wireless network and export the measured values from the sensors to the Broker. All Subscribers are been set up with Authentication to avoid unwanted access to the network and to the Broker. Several applications with graphical user interfaces (GUI) are available to interact with the parameters of the sensors. We use MyMQTT app for smartphone [8], MQTT.fx for laptop [9] with BODHI LINUX operating system and finally Node-RED [10] on WINDOWS 10 operating system in another Laptop, as shown in Fig. 4.

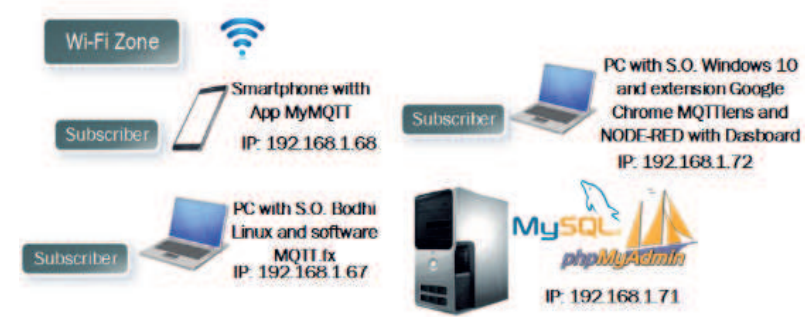

Fig. 4 Software and Hardware to subscribe and store MQTT data through a WiFi network.

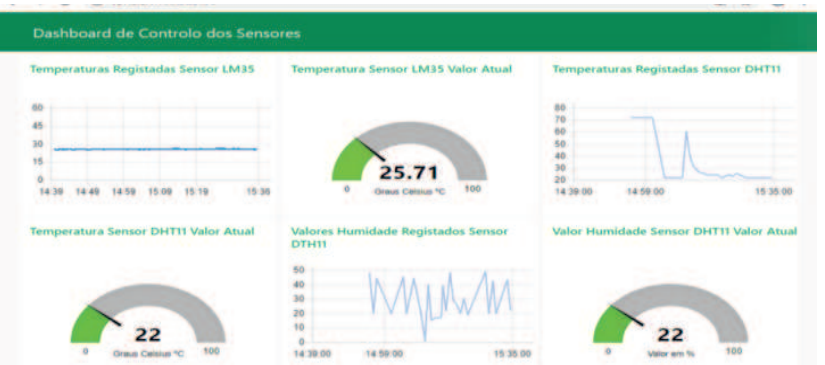

Fig. 5 Sensor monitoring dashboard with Node-RED UI.

Node-RED flow is accessible through a localhost with IP address 127.0.0.1:1880 and the corresponding dashboard is accessible through IP address 127.0.0.1:1880/ui/\#0 as can be seen in Fig. 5. With Node-RED, we can observe the sensor data and simultaneously send it to a MySQL database configured on UBUNTU Server 16.04 LTS, and then export it to PDF, Excel or CSV files.

\section{CONCLUSIONS}

The development of this demonstrator has created a affordable solution of a smartbox for industrial applications, based on the Raspberry Pi platform. It can monitor critical parameters of the shop-floor factory through open-source software, both for smartphone and Desktop / Laptop computers, as well as storing data for remote analysis. Some limitations remain to find libraries suitable to program alarm features in the OPC UA Servers. MQTT protocol is a lightweight an easy to implement M2M solution in shopfloor with limited or inexistent Ethernet network infrastructure. Future work will include additional sensors for industrial environment (vibration and angular rotation speed), alternative hardware platforms with more computational capabilities, and the implementation of Machine Learning algorithms for predictive maintenance based on the collected sensor data.

\section{REFERENCES}

[1] M. Schleipen, "OPC UA supporting the automated engineering of production monitoring and control systems", Emerging Technologies and Factory Automation 2008. ETFA 2008. IEEE International Conference, pp. 640-647, 2008.

[2] MQTT Version 3.1.1. Edited by Andrew Banks and Rahul Gupta. 29 October 2014. OASIS Standard. Retrieved from: http://docs.oasisopen.org

[3] W. Mahnke, S. H. Leitner, and M. Damm. 2009. OPC Unified Architecture (1st ed.). Springer Publishing Company, Incorporated.

[4] FreeOPCUA [Computer Software]. (2018). Retreived from: https://github.com/FreeOpcUa/python-opcua

[5] Unified Automation [Computer Software]. (2018). Retreived from: https://www.unified-automation.com/

[6] PROSYS [Computer software]. (2018). Retrieved from: https://www.prosysis.com

[7] MOSQUITTO [Computer software]. (2018). Retrieved from: https://mosquitto.org

[8] MyMQTT [Computer software]. (2018). Retrieved from: https://play.google.com

[9] MQTT.fx [Computer software]. (2018). Retrieved from: https://mqttfx.jensd.de

[10] Node-RED Programming Guide [Online]. Retrieved from: http://noderedguide.com 